\title{
Effect of corticosteroid therapy in acute pain edema caused by Herpes Zoster
}

\author{
Ruiju Zhang* and Qingguo Xu \\ Department of Dermatology, Department of Cardiology, Shandong Jiaotong Hospital, Jinan, Shandong, 250031, China
}

*For correspondence: Email: zrj150609@163.com

Received: 1 March 2016

Revised accepted: 10 July 2016

\begin{abstract}
Purpose: To evaluate the curative effect of corticosteroids in the treatment of acute pain, local edema, and skin lesions caused by herpes zoster, and to develop some pertinent therapeutic guidelines. Methods: A total of 48 cases of patients diagnosed with herpes zoster from 2010 to 2011 in the dermatology clinic of Shan Dong Traffic Hospital were selected and all received the same therapy of antiviral, pain-relieving and nerve nutrition. They were divided into a corticosteroid application group, with 24 patients treated with corticosteroids, and a control group of 24 patients without corticosteroids. Local swelling subsided in the corticosteroid group. The differences observed in pain relief and days needed for blisters to dry and scab between the two patient groups were analyzed to determine significance and, thus, assess the curative effect of corticosteroids in treatment of herpes zoster.

Results: Patients in the glucocorticosteroid application group relieved pain faster than patients in the control group ( $2.38 \pm 1.41$ days vs $5.50 \pm 3.19$ days), and the difference was significant $(p<0.05)$. Skin lesions of patients in the glucocorticosteroid application group healed quicker than that of patients in control group ( $2.83 \pm 0.87$ days vs $3.54 \pm 1.02$ days), and the difference was remarkable $(p<0.05)$. Local swelling of patients in the glucocorticosteroid application group recovered rapidly after treatment. . Conclusion: Treatment of herpes zoster with appropriate corticosteroid isodose application can effectively relieve acute pain and local swelling, and speed up scabbing and healing of skin lesions.
\end{abstract}

Keywords: Herpes zoster, Corticosteroids, Local edema, Scab, Skin lesions, Acute pain, Blisters, Therapeutic guidelines

Tropical Journal of Pharmaceutical Research is indexed by Science Citation Index (SciSearch), Scopus, International Pharmaceutical Abstract, Chemical Abstracts, Embase, Index Copernicus, EBSCO, African Index Medicus, JournalSeek, Journal Citation Reports/Science Edition, Directory of Open Access Journals (DOAJ), African Journal Online, Bioline International, Open-J-Gate and Pharmacy Abstracts

\section{INTRODUCTION}

Herpes zoster is caused by varicella-zoster virus (VZV), and is characterized by clusters of small blisters distributed along unilateral peripheral nerves and accompanied by obvious neurodynia. At present, VZV is known as human herpes virus 3 (HHV-3), is brick-shaped and has a stereoscopic and symmetrical capsid enclosing double-stranded DNA, and only one serotype has been described. Herpes zoster usually has a 2-3 weeks duration, although it may have a 3-4 weeks course in the elderly. Temporary light erythema or pigmentation is usually left after drying up and scabbing of blisters [1]. Herpes zoster is accompanied by neuralgia before rash, during rash, and after healing of skin lesions, which is called zoster-associated pain. If neuralgia continues after skin lesions subside (usually 4 weeks later), it is known as herpes zoster postherpetic neuralgia. Herpes zoster can be diagnosed according to its typical clinical manifestations. Its therapeutic principles include antiviral, pain-relieving, and anti-inflammatory treatment, and prevention of complications. Recently, it has been widely accepted that early 
and adequate anti-virus treatment can relieve neuralgia and shorten the length of the disease.

Historically, there has been a dispute over the application of corticosteroids in herpes zoster. Andrews' Diseases of the Skin [2] indicates that corticosteroids have an immunosuppressive effect, which may lead to epidemic diffusion, blood infection, and obstacles to recovery from disease. However, others recommend that corticosteroids can be used as an adjuvant therapy.

On the premise of standard antiviral therapy, through controlling inflammatory reactions, a low dose of corticosteroids in early stages [3] could reduce tissue edema, relieve pain, prevent toxic effects and destructive effects to ganglion and nerve fiber, inhibit fibrosis processes, prevent postherpetic neuralgia, and not affect production of the body's immunoglobulin (IgG) for fighting disease. A clinical trial [4] has shown that applying prednisone with acyclovir can relieve pain, promote healing of skin lesions, and shorten courses of acute neuritis so as to improve the quality of life of patients. However, it does not reduce the occurrence rate of postherpetic neuralgia.

Corticosteroids can reduce tissue edema, relieve pain, and accelerate healing of skin lesions by controlling inflammatory reactions. However, because corticosteroids can inhibit part of the body's immune function, cause disorders of water and electrolyte metabolism, and result in osteoporosis, they can be misused and infection can be induced or aggravated. Hence, corticosteroids should be selectively and cautiously applied to herpes zoster patients, and combined with adequate antiviral therapy.

At present, some propose that, on the premise of no contraindications, patients with obvious edema and acute pain in their head, face trigeminal nerve and local inflammation, who were elderly with severe pain, or with herpes zoster accompanied with local nerve dysfunction as well as Ramsay-Hunt syndrome (facial paralysis, earache, external auditory canal herpes triad) could be applied moderate-doses of corticosteroids in early stages, and the application result in improved living quality $[5,6]$.

To further examine the curative effect of corticosteroids in the treatment of herpes zoster, 24 cases of herpes zoster patients treated with corticosteroids and another 24 cases of patients treated without corticosteroids were studied.
Their pain, local edema and recovery condition of skin lesions were analyzed and statistical comparisons were made. This further exploration of the contraindication, indication, dosage and course of treatment of corticosteroids in the treatment of herpes zoster provides additional guidance to the application of corticosteroids.

\section{METHODS}

\section{Subjects}

A total of 48 cases of patients diagnosed with herpes zoster from 2010 to 2011 in the dermatology clinic of Shandong Traffic Hospital were selected. Patients were divided into a corticosteroid application group and a control group (no corticosteroid treatment) in terms of their medication. Among the 24 cases in the corticosteroid application group (male 13, female 11), all had obvious edema and acute pain in their head and face from herpes zoster and local inflammation.

Of those who had wide skin lesions, large areas of blisters and blood blisters, their pain score was above 5 . There were five patients with obvious local edema in this group. Patients had not received antiviral drugs before treatment. Patients who were severely infected or had cardiopulmonary disease as well as those with immune suppression and connective tissue diseases, with a long-term use of corticosteroids, were all excluded.

According to limiting factors such as age, rash days before hospital visit, not previously affected, underlying disease, and immunity, 24 cases (male 13, female 11) of herpes zoster patients in the control group were treated without corticosteroids. The age of patients in the two groups ranged from 33 to 85 years. All patients were informed and signed informed consents before study enrollment, and consented to the use of their samples for molecular and pathological analyses.

This clinical study was strictly carried out in accordance with the Helsinki declaration [7], and was approved by the ethical committee of Shandong Jiaotong Hospital (Research registry no. 1238).

\section{Treatment}

Patients in two groups were administered 500 $\mathrm{mg}$ of acyclovir by intravenous drip and received anti-virus twice each day. Mecobalamin was taken as 1 tablet, three times a day. Fursultimine was taken as 2 tablets, three times per day for 
trophic nerve pain. Carbamazepine was taken orally as 1 tablet, once per day to relieve pain. He-Ne laser radiation was used for irradiation for 15 min, once a day. Povidone iodine was used externally, 3 times a day. Depending on local inflammation and pain severity, the corticosteroid application group was given 2 prednisone tablets (or methylprednisolone tablets) twice or three times a day, for a 7-day course.

\section{Evaluation and scoring parameters}

The Numerical Rating Scale (NRS) was used for pain assessment. Namely, on a 0-10 pain scale, 0 represented no pain at all, 1-2 points represented mild pain, 3-4 points represented moderate pain, 5 points represented a medium degree of pain, 5-7 points represented moderate to severe pain, 8-9 points represented a serious degree of pain, and 10 points represented the most acute pain imaginable.

Substantial pain relief was defined as: [(NRS score when admitted to hospitalimmediate NRS score)/NRS score when admitted to hospital] X $100 \%$ was $\geq 50 \%$.

Local swelling was defined as subsided when: Patients made a self-assessment of swelling and determined that the local appearance had returned to normal.

Healing of skin lesions was defined as:

All blisters were dried and scabbed over completely.

\section{Evaluation methods}

The times for substantial pain relief and healing of skin lesions were recorded. Statistical analyses were conducted to determine whether there were statistical differences in times for pain relief and healing of skin lesions between the two groups. Also, the times for swelling recovery of the five patients with obvious local swelling in the corticosteroid application group were recorded. After a comprehensive analysis of the above results, the curative effect of corticosteroids in the treatment of herpes zoster was evaluated.

\section{Statistical analysis}

Data obtained were analyzed using SPSS ver. 19.0. Data conforming to normal distribution were expressed as mean \pm SD. Skew-distributed data were expressed as median. Comparison between groups was performed using analysis of variance (ANOVA) for repeated measurement and t-test. Enumeration data were compared using Chi-square test.

\section{RESULTS}

\section{Time preceding pain relief in the corticosteroid versus control group}

Seven patients in the corticosteroid group had obvious pain relief after one day of treatment. Four patients after two days and nine patients after three days had obvious pain relief. After 4, 5 and 6 days of treatment, one patient had obvious pain relief, while another had no pain relief after treatment.

One patient in the control group had pain relief after 2 days of treatment. The same results were observed for two patients after 3 days, four patients after 4 days, seven patients after 5 days, and five patients after 6 days of treatment. After $7,9,10$, and 17 days, only one patient experienced pain relief, respectively, while one patient had no pain relief after treatment. Compared with the control group, the time needed for patients to get pain relief in the corticosteroid application group was obviously shorter $(p<0.05)$. Details are shown in Figure 1. Compared with the control group, the time needed for healing of skin lesions and rash relief in glucocorticosteroid application group was shorter $(p<0.05)$. Please refer to Figure 2 and Table 1.

\section{Rash relief}

One patient in the corticosteroid application group had blisters drying up and scabbing after one day of treatment. The same result appeared in eight patients after 2 days, 9 patients after 3 days, and six patients after 4 days of treatment. One patient in the control group had blisters drying up and scabbing after one day of treatment. The same result was observed for two patients after 2 days, eight patients after 3 days, nine patients after 4 days, and four patients after 5 days of treatment. Compared with the control group, the time preceding skin lesion scabbing and healing in the corticosteroid application group was shorter $(p<0.05)$. Please refer to Figure 2 and Table 1.

\section{DISCUSSION}

Herpes zoster caused by VZV clinically presents as neuralgia and papule, blisters distributed along peripheral nerves, inflammatory response, and even local swelling when the response is severe, which has seriously influenced the quality of life in some patients [8,9]. 


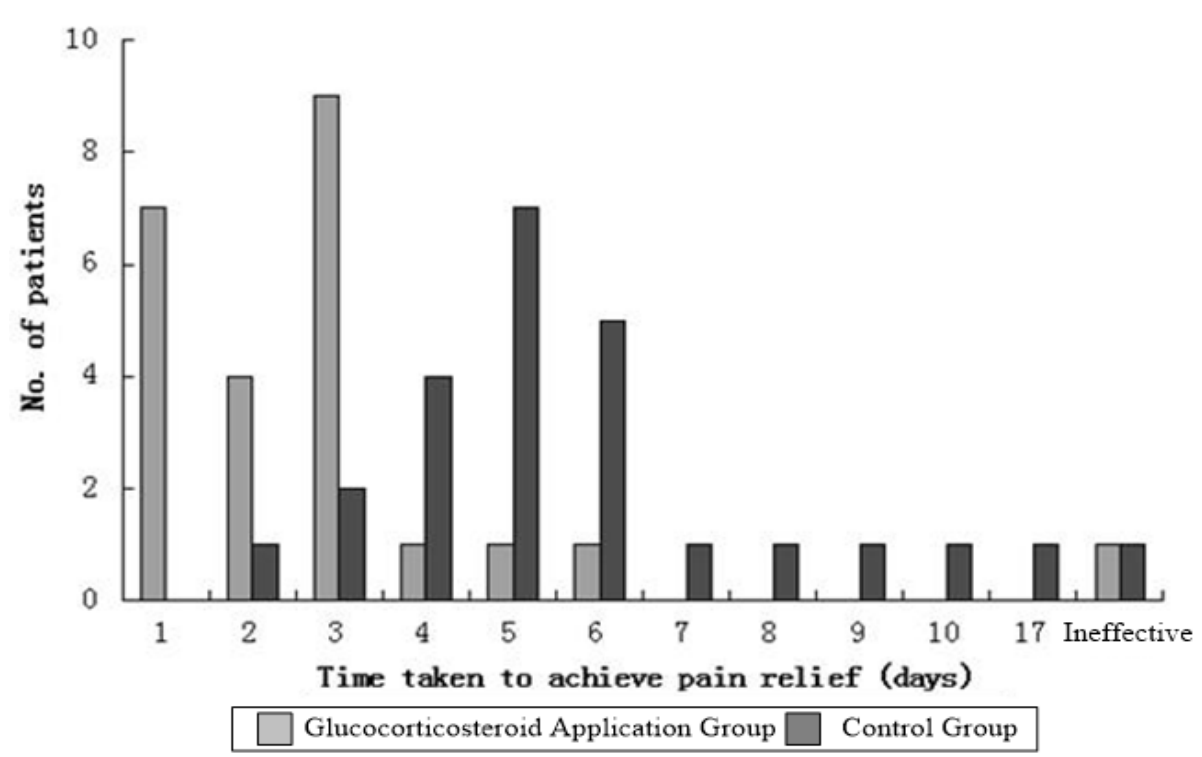

Figure 1: Time (days) preceding pain relief in the corticosteroid application and control groups

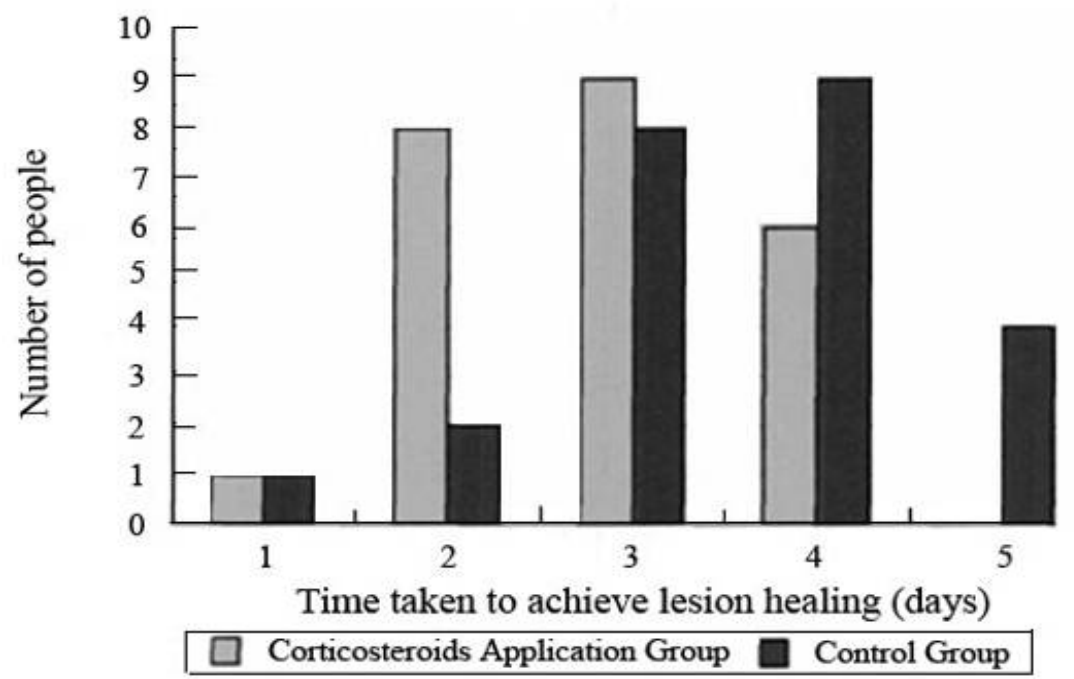

Figure 2: Rash recovery in the corticosteroid application and control groups after treatment

Table 1: Statistical data for corticosteroid curative effect on pain and skin lesions

\begin{tabular}{lccc}
\hline Group & No. of cases & $\begin{array}{c}\text { Obvious pain } \\
\text { relief(days) }\end{array}$ & $\begin{array}{c}\text { Blisters dried up } \\
\text { and scabbed (days) }\end{array}$ \\
\hline Corticosteroid & 24 & $2.38 \pm 1.41$ & $2.83 \pm 0.87$ \\
Control & 24 & $5.50 \pm 3.19$ & $3.54 \pm 1.02$ \\
Sig.(bilateral) & - & 0.000 & 0.013 \\
$P$ & - & $<0.05$ & $<0.05$ \\
\hline
\end{tabular}

Data are expressed as mean + standard deviation (SD)

Therefore, pain relief, local swelling alleviation, and local skin lesion improvement are important factors in the treatment of herpes zoster.

At present, the commonly used anti-viral drugs for treating herpes zoster are acyclovir and famciclovir, while the commonly used pain killers are carbamazepine, neurotropin, saridon, tramadol, mecobalamin, and fursultimine, which are applied for neurotrophic treatment. Some reported studies have demonstrated that combining corticosteroids with sufficient antivirus treatment can significantly relieve acute pain of herpes zoster and speed up healing of skin lesions, thus improving the quality of life of patients. However, the combinational therapy was not shown to prevent postherpetic neuralgia [10]. 
Twenty four cases of patients treated with corticosteroids and 24 cases of patients treated without corticosteroids were examined in this retrospective study. Statistical analysis of days needed for obvious pain relief, as well as skin lesion healing and scabbing, was conducted. In addition, subsiding of local swelling of five patients in the corticosteroid application group was observed, in order to confirm the curative effect of corticosteroids combined with antiretrovirus treatment of herpes zoster.

VZV infection can cause obvious inflammatory responses in local nerve fibers and the skin. Local inflammatory responses to the infection may include increased vessel permeability and leakage, tissue damage, intercellular structure damage, local swelling, and blisters [11,12]. Corticosteroids have a strong anti-inflammatory effect by inducing inflammation-inhibiting protein element 1 , and inhibiting inducible nitric oxide synthase (iNOS) and cyclooxygenase-2 (COX-2). It acts by intervening complement activation and, thus, reducing the production of inflammatory mediators; stabilizing lysosomal membranes and inhibiting the release of lysosomal enzymes, so as to alleviate tissue injury; and decrease the permeability of capillaries and inhibit infiltration and activity of phagocytic leukocytes, whereby inflammatory cellapoptosis can be induced and the release of inflammatory mediators is reduced [13]. In this way, the exosmosis of cells and body fluids can be eased, local swelling can be alleviated, and healing of skin lesions can be stimulated.

Recently, prednisone tablets have been suggested by emedicine (the world's largest original medical literature and a clinical knowledge database of Tong $\mathrm{Ji}$ reviews literature, providing doctors with a clinical decision support tool with easy operation and high authority) to take orally once a day, 10 - 50 $\mathrm{mg}$, in the treatment of herpes zoster [14].

Interactions between drugs should be considered while taking prednisone. For instance, the halflife of prednisone will increase while using it with estrogen; the risk and criticality of hypokalemia will increase while using it with digoxin and digitalis; hypnotic sedative drugs, such as dilantin and rifampicin can speed up the metabolism of prednisone; and serum potassium should be monitored while combining sodium potassium diuretics with prednisone [15]. There have been reported no common opinions about the course of corticosteroids. A course of seven days was adopted in this retrospective study and a good curative effect was achieved, so this could provide certain guidance for treatment in the future.

\section{Limitations of the study}

Patients included in the study were not followed up after treatment. Also, whether glucocorticosteroid can prevent postherpetic neuralgia is not yet known.

\section{CONCLUSION}

Neuralgia, the major clinical symptom of herpes zoster, can influence the quality of life (QoL) of patients and even induce depression and character changes. Moreover, virus infection can induce intensive inflammation on nerve and skin, increasing vascular permeability and leading to swelling. Hence, relieving pain and local swelling are of great significance in the treatment of herpes zoster. Patients with obvious edema and acute pain in their head and facial trigeminal nerve and local inflammation, elderly patients with severe pain, or patients with herpes zoster accompanied by local nerve dysfunction can be treated with a medium dose of glucocorticosteroid in the early stage, and thus improve QoL. In this study, glucocorticosteroid therapy achieved good curative effect, a finding that can guide treatment and research in future.

\section{DECLARATIONS}

\section{Acknowledgement}

The authors would like to thank Shandong Jiaotong Hospital, China and all participants for their support.

\section{Conflict of Interest}

No conflict of interest associated with this work.

\section{Contribution of Authors}

The authors declare that this work was done by the authors named in this article and all liabilities pertaining to claims relating to the content of this article will be borne by them.

\section{REFERENCES}

1. Yu XM, Wu LF. Clinical observation of applying acyclovir and prednisone in treatment of herpes zoster. Chin $\mathrm{J}$ Antibiot, 2010; 10(10): 2361-2362.

2. Parish LC. Andrews' Diseases of the Skin: Clinical Dermatology. J Am Med Assoc, 2011; 306(2): 213-213. 
3. Zhu R, Li CY, Fang M, Tang SF. Pain assessment and nursing progress of cancer patients. Med Innov Chin, 2010 7(19): 193-195.

4. Little RJ, Ralph $D$, Cohen $M L$, et al. The prevention and treatment of missing data in clinical trials. NEJM, 2012; 367(14): 1355-1360.

5. Hu PF. Clinical characteristics analysis of 89 cases of herpes zoster. J Xianning Univ: Med Sci, 2012; 26 (6): 513-514.

6. Qiu HY. Mechanism and clinical application of corticosteroids. Chin Med Mod Dist Edu Chin, 2010; 11(4): 90-91.

7. Mellin-Olsen J, Staender S. The Helsinki Declaration on Patient Safety in Anaesthesiology: the past, present and future.[J]. Current Opinion in Anaesthesiology, 2010, 27(6):630-634.

8. Lu ZJ, Ma ZM. Oral complication and nursing of patients with long time inhalation of corticosteroids. Chin J Prim Med Pharm, 2012; 19(9): 1416-1417.

9. Wang WL. Clinical analysis of famciclovir with corticosteroids in treatment of herpes zoster in old man. Chin J Postgrad Med, 2009; 32(30): 34-35.
10. Gan EY, Tian EAL, Hong LT. Management of Herpes Zoster and Post-Herpetic Neuralgia. Am J Clin Dermatol, 2013; 14(2): 77-85.

11. Yuan HG, Chen D. Curative effect of corticosteroids in treating with herpes zoster. Chin J Dermatovenerol Integr Trad W Med, 2011; 10(6): 378-379.

12. Ran XP, Bi P. Clinical analysis of 64 cases of diabetes complicated with multiple organ failure. Chin J Prim Med Pharm, 2012; 19(18): 2756-2757.

13. Hulce JJ, Cognetta AB, Niphakis MJ, Tully SE, Cravatt $B F$. Proteome-wide mapping of cholesterol-interacting proteins in mammalian cells. Nat Meth, 2013; 10(3): 259-264.

14. Chen SW. Comparative study on application of glucocorticoids system in treating herpes zoster. Chin Med Pharm, 2012; 2(18):235-236.

15. Marik PE, Meduri GU, Rocco PR, Annane D. Glueocorticoid treatment in acute lung injury and acute respiratory distress syndrome. Crit Care Clin, 2011; 27(3): 589-607. 